\title{
Laparoscopic ventral hernia repair with new generation bilaminar mesh
}

\author{
Başar Aksoy, ${ }^{1}$ Erdal Uysal, ${ }^{1}$ M. Fatih Yüzbaşıŏlu, ${ }^{1}$ Ahmet Orhan Gürer, ${ }^{1}$ \\ Hasan Bakır, ${ }^{1}$ Mehmet Ali İkidağ ${ }^{2}$ \\ 'Department of General Surgery, Sanko University Faculty of Medicine, Gaziantep, Turkey \\ ${ }^{2}$ Department of Radiology, Sanko University Faculty of Medicine, Gaziantep, Turkey
}

\begin{abstract}
Introduction: The aim of this study was to assess laparoscopic ventral hernia repair (LVHR) using new generation bilaminar mesh and to share our experience. Laparoscopic approaches are preferred in the surgical repair of ventral hernia due to low postoperative pain and complication rates, quick recovery, and short time for return to work. They can be performed in primary ventral hernia as well as incisional hernia, which is one of the most frequent complications of surgical procedures. Furthermore, recurrence rate is low, unlike open-conventional hernia repair.

Materials and Methods: Fifteen patients who underwent laparoscopic ventral-incisional hernia repair between 2012 and 2014 were included in the study. A patch, 20x15 cm in size, of Parietex (Covidien-Medtronic, Inc. Minneapolis, MN, USA) polyester mesh, which has absorbable collagen film and preplaced sutures, was used. Mesh was fixed to the abdominal wall with transfascial fixation sutures and tacker. Patients were periodically followed-up for evaluation of hernia recurrence, pain, infection, and other complications.

Results: Fifteen patients ( 3 male and 12 female) underwent laparoscopic ventral hernia repair. Mean operating time was 118.75 minutes (range: 120-250 minutes). Mean length of hospitalization was 2.8 days. One patient had small intestine laceration during surgery. One patient had hernia recurrence, 1 developed seroma, and 1 experienced prolonged pain.

Conclusion: LVHR is an efficient and reliable method of ventral hernia repair. It is easy to perform in midline small and average-sized defects. It is possible to perform successful operation with low complication rate using new generation bilaminar mesh. In order to avoid prolonged postoperative pain, redundant use of tacker should be avoided. Transfascial fixation sutures will reduce recurrence rate.

Keywords: Bilaminar meshes; laparoscopic ventral hernia.
\end{abstract}

\section{Introduction}

Since its first introduction in 1993, laparoscopic ventral hernia repairments (LVHR) have been widely used and are getting more popular due to quick recovery time, low postoperative pain, low complication rates and short time in returning to work. ${ }^{[1]}$ It can be used in both primary ventral hernias and incisional hernias, which still remain one 
of the most significant complications of common surgical procedures. Unlike openconventional hernia repairments, wide dissections are not necessary and recurrence rates are low. ${ }^{[2,3]}$

Moreover, with the introduction of the use of bilaminar meshes, risk of significant complications such as adhesions, erosions and fistulas are diminished. ${ }^{[3,4]}$ Numerous kinds of synthetic patches are available, but today bilaminar-laminated polypropylene, polyester meshes are mostly used. The inner-visceral side of these meshes are covered with a thin layer of expanded polytetrafl uoroethylene (ePTFE), collagen or seraphim in order to prevent adhesions. ${ }^{[5]}$

The aim of this study was to assess the use of laparoscopic ventral hernia repairments (LVHR) by bilaminary new generation meshes and share our experience.

\section{Materials and Methods}

Fifteen patients who underwent laparoscopic ventral-incisional hernia repairment between 2012 and 2014 were included into the study. The size and locations of the hernias and the presence of intestinal loops were assessed by CT. Demographic knowledge and medical history, such as previous abdominal surgery, were achieved from hospital records and information system retrospectively (Table 1).

A 20x15 cm Parietex (optimized composite mesh, Covidien, France) polyester mesh, having absorbable collagen film and preplaced sutures, was used. Patients received general anesthesia in supine position. Prophylactic IV cefazolin $1 \mathrm{~g}$ was administered at the beginning of anesthesia. Orogastric cannula was placed to maintain gastric decompression. Three trocars were used during surgery; one $12 \mathrm{~mm}$ trocar was placed lateral to rectus sheath and two $5 \mathrm{~mm}$ trocars were placed neighboring superior and inferior to the first one. $30^{\circ}$ laparoscopy camera was usedunder $13 \mathrm{mmHg}$ pressure.

Omental and intestinal adhesions were separated by monopolar diathermy, harmonic scalpel or scissors, and wide abdominal view was achieved. Vessel Sealing system was used to control bleedings and in dissections. Defect margins were delineated by spinal needles and abdominal wall was marked. Parietex mesh, which had four-sided preplaced propylene sutures, was rolled and sent into the abdominal cavity through a $12 \mathrm{~mm}$ trocar, and then opened and restored inside the cavity as the upper side would cover the defect. Polypropylene surface was directly placed on the abdominal wall. Corner sutures were taken out of the abdomen by suture taking instrument and tied-connected-attached over the fascia by a small incision. Mesh was fixated to the abdominal wall attaching metal tacker (Ethicon). After last control and hemostasis, abdominal carbon dioxide was evacuated and the operation was terminated. Drainage catheters were not used.

Patients were periodically controlled for the evaluation of hernia recurrence, pain, infection and other complications.

\section{Table 1. Age, gender, and sizes of the patients}

\begin{tabular}{llccc}
\hline Age & Gender & Defect size & Hernia location & Primary/secondary hernia \\
\hline 59 & Female & $3-4 \mathrm{~cm}$ & Epigastric + umbilical & Seconder \\
74 & Female & $4 \mathrm{~cm}$ & Umbilical & Primer \\
50 & Female & $4 \mathrm{~cm}$ & Epigastric & Seconder \\
66 & Female & $5 \mathrm{~cm}$ & Epigastric & Primer \\
55 & Male & $3 \mathrm{~cm}$ & Epigastric & Primer \\
50 & Female & $6 \mathrm{~cm}$ & Midline & Seconder \\
61 & Female & $7 \mathrm{~cm}$ & Umbilical & Primer \\
62 & Female & $3 \mathrm{~cm}$ & Umbilical & Primer \\
54 & Female & $6 \mathrm{~cm}$ & Right inguinal & Seconder \\
41 & Female & $3 \mathrm{~cm}$ & Umbilical & Primer \\
45 & Male & $5 \mathrm{~cm}$ & Midline & Seconder \\
60 & Female & $4 \mathrm{~cm}$ & Umbilical & Seconder \\
61 & Female & $5 \mathrm{~cm}$ & Midline & Primer \\
55 & Female & $5 \mathrm{~cm}$ & Right subcostal & Seconder \\
51 & Male & $10 \mathrm{~cm}$ & Left subcostal & Seconder \\
\hline
\end{tabular}




\section{Table 2. Complications}

\begin{tabular}{ll}
\hline Complications number of patient & \\
\hline Prolonged pain & 1 \\
Seroma & 1 \\
Bowel laceration & 1 \\
Recurrence & 1
\end{tabular}

\section{Results}

Fifteen patients ( 3 male and 12 female) underwent laparoscopic ventral hernia repairment. Mean operation duration was $118.75 \mathrm{~min}$ (range, 120-250 min). Mean hospitalization time was 2.8 days. Patients were able to return to their jobs and daily routines approximately on the fifteenth day. Parenteral analgesics were administered only on the first three days of the operation, except for one patient who had prolonged severe abdominal pain lasting for nearly one month. One patient was operated for incisional hernia had seroma, which was drained by a syringe. Another patient had a recurrent ventral hernia and was admitted to the emergency department two months after surgery by incarcerated recurrent ventral hernia. This patient underwent urgent open ventral hernia repairment. The largest defect size was $10 \mathrm{~cm}$ and the smallest was 3.

Small intestine segments were observed in the hernia sac in a patient with incisional hernia, who had a history of gastric bypass surgery. Dissection was hardly made due to severe adhesions, multiple lacerations occurred in this segment, and extracorporeal resection anastomosis was performed. There was no need to convert to open procedure in any patient. There were no other complications regarding anesthesia or surgery. The complications encountered in our patients are listed in Table 2.

\section{Discussion}

As minimally invasive surgical procedures are popular today, laparoscopic ventral hernia repairments can also be reliably performed. Open surgical repairments and laparoscopic ones have been reported to have similar recurrence rates as some investigators found lower recurrence by laparoscopic surgery. ${ }^{[3,6-8]}$

By using new generation bilaminar meshes, important complications like fistulas are significantly reduced, therefore appropriate mesh selection is substantial. None of our patients developed fistulas.
Pain is an important problem after LVHR. Postoperative pain causes delay in intestinal functions and leads to prolonged hospitalization. ${ }^{[9]}$ It has been reported that chronic pain after LVHR occurrence rate is $1-3 \% .^{[7-10]}$ In one of our patients, operated for right subcostal and midline incisional hernia, prolonged abdominal pain developed that lasted forty-five days and responded partially to medications. It was thought that this severe and prolonged pain was due to irritation of costal periostitis by the tacker during fixation of the mesh to the abdominal wall. Jenkins et al. have reported that chronical pain due to tacker is $7.4 \% .{ }^{[10]}$

Selection of fixation material and adequate fixation of the mesh to the abdominal wall are important parameters effecting the success rates of LVHR. There are numerous kinds of fixation materials available for this surgery. Mostly, metal tackers and non-absorbable suture materials are used. Metallic non-absorbable tackers may cause life threatening complications like bowel perforation, if not used carefully. ${ }^{[1,11]}$ In addition, tackers should not be dropped into the abdominal cavity. Adequate fixation of the tackers to the abdominal wall must be ensured. None of our patients developed bowel perforation due to tackers.

Hernia recurrence is one the most common problems following LVHR. The major causes leading recurrences are inadequate fixation, strained repairment, using tacker without transfascial abdominal fixation sutures, migration, and protrusion of mesh due to technical problems in fixations. One of our patients developed early recurrent hernia, in whom transfascial fixation sutures were not used. In all other patients, transfascial propylene sutures were uses, which were present at four sides of the mesh. ${ }^{[1]}$

Hernia sacs were not resected from the abdominal wall. Furthermore, hernia defects were not sutured. The patients were advised to use elastical abdominal corset during mobilization for six months. Emphasis is given on the mesh to be larger than the defect sizefor tension free repairment. Recurrence rates are known to be higher in non-tension free repairments.

Quick recovery time and short time in returning to work are important parameters regarding the success and feasibility of a surgical procedure. Like other laparoscopic procedures, patients who underwent LVHR can return to their daily routines and works earlier, as they have lower pain, quick recovery and low complication rates. 
LVHR is an efficient and reliable ventral hernia repairment method. It is easy to perform in midline, small and average size defects. It is possible to perform successful operations with low complication rates by new generation bilaminar mashes. In order to avoid prolonged postoperative pain, redundant use of tacker should be avoided. Transfascial fixation sutures will reduce recurrence rates.

\section{References}

1. Cavallaro G, Campanile F, Rizzello M, Greco F, Iorio O, Angelis FD, et al. Laparoscopic incisional hernia repair by lightweight polypropylene mesh with resorbable coating. Technical notes, preliminary results. Chirurgia 2013;108:304-11.

2. Bhanot P, Franklin BR, Patel KM. Proceed ${ }^{\mathrm{TM}}$ mesh for laparoscopic ventral hernia repair. JSLS 2013;17:565-9.

3. Heniford BT, Park A, Ramshaw BJ, Voeller G. Laparoscopic repair of ventral hernias: nine years' experience with 850 consecutive hernias. Ann Surg 2003;238:391-9.

4. Cobb WS, Kercher KW, Heniford BT. The argument for lightweight polypropylene mesh in hernia repair. Surg Innov 2005;12:63-9.

5. Torres-Villalobos G, Sorcic L, Ruth GR, Andrade R, Martin-del-Campo LA, Anderson JK. Evaluation of the Rebound
Hernia Repair device for laparoscopic hernia repair. JSLS 2010;14:95-102.

6. Sauerland S, Walgenbach M, Habermalz B, Seiler CM, Miserez M. Laparoscopic versus open surgical techniques for ventral or incisional hernia repair. Cochrane Database Syst Rev 2011:CD007781.

7. Grubnik VV, Grubnik AV, Vorotyntseva KO. Laparoscopic repair of incisional and ventral hernias with the new type of meshes: randomized control trial. Wideochir Inne Tech Maloinwazyjne 2014;9:145-51.

8. Berger D, Bientzle M, Müller A. Postoperative complications after laparoscopic incisional hernia repair. Incidence and treatment. Surg Endosc 2002;16:1720-3.

9. Bellows $\mathrm{CF}$, Berger DH. Infiltration of suture sites with local anesthesia for management of pain following laparoscopic ventral hernia repairs: a prospective randomized trial. JSLS 2006;10:345-50.

10. Jenkins ED, Yom V, Melman L, Brunt LM, Eagon JC, Frisella $M M$, et al. Prospective evaluation of adhesion characteristics to intraperitoneal mesh and adhesiolysis-related complications during laparoscopic re-exploration after prior ventral hernia repair. Surg Endosc 2010;24:3002-7.

11. Sasse KC, Lim DC, Brandt J. Long-term durability and comfort of laparoscopic ventral hernia repair. JSLS 2012;16:3806. 